\title{
L-657,398, A NOVEL ANTIFUNGAL AGENT: FERMENTATION, ISOLATION, STRUCTURAL ELUCIDATION AND BIOLOGICAL PROPERTIES
}

\author{
Robert E. Schwartz, Jerrold Liesch, Otto Hensens, Lauretta Zitano, \\ Sue Honeycutt, George Garrity, Robert A. Fromtling, \\ JANET ONISHI and RICHARD MONAGHAN \\ Merck Sharp \& Dohme Research Laboratories, \\ P. O. 2000 (R 80Y 3D-340) Rahway, N.J., U.S.A.
}

(Received for publication June 23, 1988)

\begin{abstract}
L-657,398 is a broad spectrum antifungal agent isolated from solid fermentation or from the mycelium of the liquid fermentation of Aspergillus ochraceus. Structurally, the compound is a novel pyrollidine related to anisomycin.
\end{abstract}

Various isolates of Aspergillus ochraceus produce ochratoxin, xanthomegnin, mellein, vionellein, trypacidin, aspyrone, ergosta-4,6,8(14),22-tetraene-3-one, flavacol, penicillic acid and two modified asperigillic acids ${ }^{12}$. The subject of this paper is the production of a new secondary metabolite, the pyrollidine L-657,398 (1), from the fermentation of A. ochraceus ATCC 22947. L-657,398 is structurally related to anisomycin (3), an anti-protozoan and anti-yeast compound isolated from Streptomyces griseolus and Streptomyces roseochromogenes ${ }^{2 \sim 4)}$. Anisomycin is reported to be a potent and reversible inhibitor of protein synthesis in certain yeasts and mammalian cells ${ }^{5)}$. The fermentation, isolation, structural elucidation and antifungal activity of L-657,398 are reported in this paper.

\section{Materials and Methods}

Isolations and fermentations were monitored initially via zones of inhibition in an agar-disc diffusion assay using Candida albicans (MY992, Table 3) and later with TLC, E. Merck, Kieselgel $60 \mathrm{~F}_{254}$, EtOAc, Rf 0.21, iodine visualization.

Flask Fermentation

The seed medium for growth of $A$. ochraceus in flasks consisted of corn steep liquor $5.0 \mathrm{~g} / \mathrm{liter}$, tomato paste $40.0 \mathrm{~g} /$ liter, oat flour $10.0 \mathrm{~g} /$ liter, glucose $10.0 \mathrm{~g} /$ liter and trace element mix $10 \mathrm{ml} /$ liter. Trace element mix contained $\mathrm{FeSO}_{4} \cdot 7 \mathrm{H}_{2} \mathrm{O} 1 \mathrm{~g} /$ liter, $\mathrm{MnSO}_{4} \cdot 4 \mathrm{H}_{2} \mathrm{O} 1 \mathrm{~g} / \mathrm{liter}, \mathrm{CuCl}_{2} \cdot 2 \mathrm{H}_{2} \mathrm{O} 25 \mathrm{mg} / \mathrm{liter}$, $\mathrm{CaCl}_{2} 100 \mathrm{mg} / \mathrm{liter}, \mathrm{H}_{3} \mathrm{BO}_{3} 56 \mathrm{mg} /$ liter, $\left(\mathrm{NH}_{4}\right)_{8} \mathrm{Mo}_{7} \mathrm{O}_{24} \cdot 4 \mathrm{H}_{2} \mathrm{O} 19 \mathrm{mg} /$ liter and $\mathrm{ZnSO}{ }_{4} \cdot 7 \mathrm{H}_{2} \mathrm{O} 200 \mathrm{mg} /$ liter. The medium was adjusted to $\mathrm{pH} 6.8$ with $\mathrm{NaOH}$ prior to sterilization. A. ochraceus ATCC 22947 was inoculated into $50 \mathrm{ml}$ of this sterilized growth medium and cultured for 3 days on a rotary shaker ( $212 \mathrm{rpm}, 5 \mathrm{~cm}$ throw) at $28^{\circ} \mathrm{C}$.

Two $\mathrm{ml}$ of the seed medium culture was transferred, after growth, to a 250 -ml unbaffled Erlenmeyer flask containing of cracked corn $10.0 \mathrm{~g}$, yeast hydrolysate (Ardamine pH) $2.0 \mathrm{mg}, \mathrm{KH}_{2} \mathrm{PO}_{4}$ $1.0 \mathrm{mg}, \mathrm{MgSO}_{4} \cdot 7 \mathrm{H}_{2} \mathrm{O} 1.0 \mathrm{mg}$, sodium tartrate $1.0 \mathrm{mg}, \mathrm{FeSO}_{4} \cdot 7 \mathrm{H}_{2} \mathrm{O} 0.1 \mathrm{mg}, \mathrm{ZnSO}_{4} \cdot \mathrm{H}_{2} \mathrm{O} 0.1 \mathrm{mg}$ and $25.0 \mathrm{ml}$ of distilled water. After inoculation flasks were incubated for 14 days on a rotary shaker (220 rpm, $5 \mathrm{~cm}$ throw) at $25^{\circ} \mathrm{C}$.

\section{Fermentor Fermentation}

The constituents of the seed medium for the fermentor fermentation were the same as the seed medium described for the flask fermentation above. A lyophilized culture of A. ochraceus ATCC 
22947, was used to inoculate a $250-\mathrm{ml}$ Erlenmeyer flask containing $50 \mathrm{ml}$ of seed medium. This seed flask was incubated at $28^{\circ} \mathrm{C}$ for 48 hours on a rotary shaker at $220 \mathrm{rpm}$. Two \% inoculum was used to inoculate 2-liter unbaffled Erlenmeyer flasks containing $500 \mathrm{ml}$ of the seed medium previously described. The second stage seed flasks were incubated at $28^{\circ} \mathrm{C}$ for 24 hours on a rotary shaker at $200 \mathrm{rpm}$.

The production medium was adjusted to $\mathrm{pH} 7.0$ prior to sterilization in a nutrient sterilizer. Four sterile New Brunswick fermentors were charged with sterile medium consisting of glucose $10.0 \mathrm{~g} / \mathrm{liter}$, glycerol $10.0 \mathrm{~g} /$ liter, corn steep liquor $5.0 \mathrm{~g} /$ liter, $\left(\mathrm{NH}_{4}\right)_{2} \mathrm{SO}_{4} 2.0 \mathrm{~g} /$ liter, corn meal $10.0 \mathrm{~g} /$ liter, $\mathrm{CoCl}{ }_{2}$. $6 \mathrm{H}_{2} \mathrm{O} 10.0 \mathrm{mg} /$ liter, soybean meal $5.0 \mathrm{~g} /$ liter, glycine $2.0 \mathrm{~g} /$ liter and $\mathrm{P}-2000$ antifoam (Dow) $3.0 \mathrm{ml} /$ liter in distilled water. Five \% inoculum was then used to inoculate each 14-liter scale fermentor containing 10 liters of production medium. The fermentations were carried out at $28^{\circ} \mathrm{C}$ under a range of conditions for airflow ( 2.0 to 5.0 liters/minute) and agitation rate (200 to $500 \mathrm{rpm}$ ) for 90 hours.

\section{Isolation Flask Fermentation}

The solid fungal fermentation consisted of thirty-four 250-ml flasks (initially containing $35 \mathrm{~g}$ of corn based medium per flask) which were extracted by adding $100 \mathrm{ml}$ of $\mathrm{MeOH}$, stirring to break up the fungal/medium cake, steeping overnight at room temperature and filtering to yield $2,600 \mathrm{ml}$ of filtrate. The spent mycelium was extracted again with $\mathrm{MeOH}$ and filtered to yield 2,500 $\mathrm{ml}$ of filtrate.

The first extraction $(2,600 \mathrm{ml})$ was partitioned into two layers by adding $650 \mathrm{ml}$ of water and $3,250 \mathrm{ml}$ of $\mathrm{CH}_{2} \mathrm{Cl}_{2}$ to make the mixture $4: 1: 5, \mathrm{MeOH}$ extract $-\mathrm{H}_{2} \mathrm{O}-\mathrm{CH}_{2} \mathrm{Cl}_{2}$. The lower $\mathrm{CH}_{2} \mathrm{Cl}_{2}$ $\mathrm{MeOH}$ layer $(3,300 \mathrm{ml})$, contained all the antifungal activity and only $20 \%$ of the solids, while the upper $\mathrm{MeOH}$ layer is $3,050 \mathrm{ml}$. A similar procedure was performed on the second $\mathrm{MeOH}$ extract $(2,500 \mathrm{ml})$.

The two $\mathrm{CH}_{2} \mathrm{Cl}_{2}-\mathrm{MeOH}$ lower layers from the partitioning steps described above were combined, concentrated to dryness and taken up in $100 \mathrm{ml}$ of EtOAc. This concentrate was chromatographed on 1 liter of silica gel (E. Merck, Grade 62, 60 200 mesh) using EtOAc as the eluant, and the rich cuts from this chromatography were rechromatographed on $200 \mathrm{ml}$ silica gel (EM Science, Kieselgel $60,230 \sim 400$ mesh) using EtOAc as the eluting solvent.

The rich cuts from the second silica gel chromatography were combined, concentrated to dryness, diluted to $2 \mathrm{ml}$ with hexane $-\mathrm{CH}_{2} \mathrm{Cl}_{2}-\mathrm{MeOH}(10: 10: 1)$ and chromatographed on $100 \mathrm{ml}$ Sephadex LH-20 in the same solvent system. The rich cuts from this chromatography were combined, concentrated and weighed to give $18.9 \mathrm{mg}$ of pure L-657,398 (1).

\section{Isolation Fermentor Fermentation}

Four 14-liter scale fermentations of $A$. ocraceus ATCC 22947 were filtered and the mycelia extracted with 2 liters $\mathrm{CH}_{2} \mathrm{Cl}_{2}-\mathrm{MeOH}(1: 1)(2 \times)$ and 1 liter $\mathrm{CH}_{2} \mathrm{Cl}_{2}-\mathrm{MeOH}(1: 1)(1 \times)$. The first extraction yielded two layers due to the water present in the mycelium. The lower $\mathrm{CH}_{2} \mathrm{Cl}_{2}-\mathrm{MeOH}$ layer contained L-657,398. The second 2 liters extraction and third 1 liter extraction resulted in a single phase, but the addition of $200 \mathrm{ml}$ of $\mathrm{H}_{2} \mathrm{O}$ to each extract resulted in two layers, the lower $\left(\mathrm{CH}_{2} \mathrm{Cl}_{2}\right.$ $\mathrm{MeOH}$ ) of which, again, contained $\mathrm{L}-657,398$. All the $\mathrm{CH}_{2} \mathrm{Cl}_{2}-\mathrm{MeOH}$ layers were combined, concentrated to a small volume and rediluted to $500 \mathrm{ml}$ with EtOAc.

This concentrate was chromatographed on 5 liters of silica gel (E. Merck, Grade 62, $60 \sim 200$ mesh) using EtOAc as the eluting solvent and a flow rate of $100 \mathrm{ml} /$ minute. Active fractions, detected via antifungal bioassay, were combined, concentrated to $60 \mathrm{ml}$ in vacuo and diluted to $200 \mathrm{ml}$ with $\mathrm{CH}_{2} \mathrm{Cl}_{2}$ hexane- $\mathrm{MeOH}(10: 10: 1)$. This preparation was then chromatographed on 1.5 liters Sephadex LH-20 in the same $\mathrm{CH}_{2} \mathrm{Cl}_{2}$ - hexane - $\mathrm{MeOH}(10: 10: 1)$ solvent system.

The rich cut from the Sephadex LH-20 separation was then chromatographed on 2 liters silica gel (E. Merck, Grade 62, 60 200 mesh) using a step gradient of EtOAc - hexane (2:8) followed by EtOAc - hexane $(1: 1)$ and a flow rate of $30 \mathrm{ml} / \mathrm{minute}$. The active fractions were then combined, concentrated and chromatographed on $200 \mathrm{ml}$ silica gel (EM Science, Kieselgel 60, 230 400 mesh) using a step gradient of EtOAc - hexane $(35: 65)$ followed by EtOAc - hexane $(1: 1)$ and a flow rate of $4 \mathrm{ml} /$ minute. In both cases the active constituent eluted with the EtOAc-hexane $(1: 1)$ solvent system. 
The final purification step was chromatography on $200 \mathrm{ml}$ Sephadex $\mathrm{LH}-20 \mathrm{CH}_{2} \mathrm{Cl}_{2}$ - hexane $\mathrm{MeOH}(10: 10: 1)$ with a flow rate of $2 \mathrm{ml} /$ minute to yield, upon concentration, $619 \mathrm{mg}$ of $\mathrm{L}-657,398$ (1) as a waxy solid.

\section{Spectroscopic Methods}

${ }^{1} \mathrm{H}$ and ${ }^{13} \mathrm{C}$ NMR spectra were obtained at 400 and $100 \mathrm{MHz}$, respectively, on a Varian XL400 spectrometer at ambient room temperature. Electron impact mass spectra (EI-MS) data were obtained on a Finnigan MAT-212 mass spectrometer; fast atom bombardment (FAB) data were obtained on a Finnigan MAT-731 mass spectrometer. UV spectra were obtained on a Beckman DU70 spectrophotometer.

\section{L-657,398 (1)}

The molecular weight of L-657,398 was determined to be 317 by FAB-MS (observed $(\mathrm{M}+\mathrm{H})^{+}$ at $\mathrm{m} / z$ 318). However, EI-MS of L-657,398 disclosed an apparent molecular ion at $\mathrm{m} / \mathrm{z} 316$ which corresponds to $(\mathrm{M}-\mathrm{H})^{+}$. Abundant $(\mathrm{M}-\mathrm{H})^{+}$ions are frequently observed in cyclic amines under EI-MS conditions ${ }^{6)}$. The molecular formula $\mathrm{C}_{23} \mathrm{H}_{35} \mathrm{NO}$ (calcd for $\mathrm{C}_{21} \mathrm{H}_{34} \mathrm{NO} 316.2639$, found 316.2635, $\left.(\mathrm{M}-\mathrm{H})^{+}\right)$was assigned to $\mathrm{L}-657,398$ based upon the FAB and EI data.

The UV spectrum in $\mathrm{MeOH}$ showed a peak at $203 \mathrm{~nm}, \mathrm{E}_{1 \mathrm{~cm}}^{1 \%} 407 .{ }^{13} \mathrm{C}$ and ${ }^{~} \mathrm{H}$ NMR data are listed in Tables 1 and 2, respectively, of the Results and Discussion section.

\section{L-657,398 Acetate (2)}

L-657,398, $16.6 \mathrm{mg}$, was dissolved in pyridine and acetic anhydride was added at room temperature to produce the monoacetate derivative, $2\left(16.8 \mathrm{mg}\right.$ ), of L-657,398. $\mathrm{C}_{23} \mathrm{H}_{37} \mathrm{NO}_{2}$ (calcd $m / z$ 358.2746, found $\mathrm{m} / \mathrm{z} 358.2705$, for $\mathrm{M}^{+}-\mathrm{H}$ ) via high resolution (HR)-MS. ${ }^{13} \mathrm{C} \mathrm{NMR}$ data for this compound is reported in Table 1 of the Results and Discussion section.

\section{Anisomycin (3)}

Authentic anisomycin was obtained from Sigma, St. Louis, Mo., catalog number A 9789, lot number 56F-4026.

\section{Antifungal Assays}

The microbial strains used are listed in Table 3. Stock cultures of filamentous fungi were maintained on potato dextrose agar (Difco, Detroit, Mich.) and were transferred serially at 2 weeks intervals using standard microbiological techniques. Stock yeast strains, Streptomyces sp. (MA4798) and Acholeplasma laidlawii (MB4558) were maintained frozen at $-80^{\circ} \mathrm{C}$ in $20 \%$ aqueous glycerol.

Seeded agar assay plates were prepared according to the type of assay strain. Inoculum for filamentous fungi was prepared by scraping the surface of stock plates with a moistened sterile dacron swab. The spores and mycelia were then suspended in $10 \mathrm{ml}$ of sterile potato dextrose broth (PDB) and adjusted to $70 \%$ transmittance $(\mathrm{T})$ at $660 \mathrm{~nm}$. Inoculum for yeasts and bacterial strains was prepared from overnight broth cultures. With the exception of $A$. laidlawii, which required specialized preparation, cultures were then diluted into PDB to a final concentration of either 40 or $70 \% \mathrm{~T}$ at $660 \mathrm{~nm}$ (see Table 3). A. laidlawii was suspended in brain heart infusion broth supplemented with $2.5 \%$ yeast extract and $20 \%$ heat inactivated horse serum and adjusted to a final concentration of $60 \%$ $\mathrm{T}$ at $660 \mathrm{~nm}$. Assay plates were prepared by diluting the inoculum into appropriate molten agar medium, cooled to $45^{\circ} \mathrm{C}$, to yield a final concentration of $4 \%$.

Samples were applied to $6.2 \mathrm{~mm}$ filter paper discs $(25 \mu \mathrm{l} / \mathrm{disc})$ and air dried at $24^{\circ} \mathrm{C}$. The discs were then applied to seeded assay plates with sterile forceps, and rewetted with $25 \%$ sterile aq DMSO. The assay plates were then incubated at either 28 or $37^{\circ} \mathrm{C}$ for 24 hours (see Table 3).

Following incubation, inhibition zones were measured and recorded. Measurement was from the extreme edge of any zone where the growth differs from the background lawn. Inhibition zones were further qualified as follows: fuzzy (F) - a zone that had a fuzzy edge and clear center surrounding the disc, hazy $(\mathrm{H})$ - a zone that was hazy throughout, slightly hazy $(\mathrm{S})$ - a zone in which low levels of growth were discernible throughout the inhibition zone, and very hazy (V) - a zone in which the differences between the background lawn and inhibition zone were barely discernable. Zones without a qualifier were clear throughout. 
Table 1. ${ }^{13} \mathrm{C}$ NMR assignments of L-657,398 (1) and acetate $(2)^{\mathrm{a}}$.

\begin{tabular}{lccc}
\hline Assignment & $1^{\mathrm{b}}$ & $\mathbf{1}^{\mathrm{c}}$ & $\mathbf{2}^{\mathrm{b}}$ \\
\hline $\mathrm{C}-14$ & 14.1 & $14.4 \mathrm{q}$ & $14.1 \mathrm{q}$ \\
$\mathrm{COCH}$ & & & $21.3 \mathrm{q}$ \\
$\mathrm{C}-13$ & $22.7 \mathrm{t}$ & $23.5 \mathrm{t}$ & $22.7 \mathrm{t}$ \\
$\mathrm{C}-7$ & $26.3 \mathrm{t}$ & 27.1 & $26.5 \mathrm{t}$ \\
$\mathrm{C}-8^{\mathrm{d}}$ & $29.3 \mathrm{t}$ & $30.2 \mathrm{t}$ & $29.3 \mathrm{t}$ \\
$\mathrm{C}-9^{\mathrm{d}}$ & $29.58 \mathrm{t}$ & $30.2 \mathrm{t}$ & $29.5 \mathrm{t}$ \\
$\mathrm{C}-10^{\mathrm{d}}$ & $29.65 \mathrm{t}$ & $30.3 \mathrm{t}$ & $29.6 \mathrm{t}$ \\
$\mathrm{C}-11^{\mathrm{d}}$ & $29.9 \mathrm{t}$ & $30.4 \mathrm{t}$ & $29.7 \mathrm{t}$ \\
$\mathrm{C}-1$ & $31.9 \mathrm{t}$ & $31.2 \mathrm{t}$ & $31.8 \mathrm{t}$ \\
$\mathrm{C}-6$ & $33.6 \mathrm{t}$ & $32.2 \mathrm{t}$ & $33.9 \mathrm{t}$ \\
$\mathrm{C}-12$ & $34.8 \mathrm{t}$ & $32.8 \mathrm{t}$ & $34.6 \mathrm{t}$ \\
$\mathrm{NCH}$ & $38.6 \mathrm{q}$ & $33.8 \mathrm{q}$ & $37.9 \mathrm{q}$ \\
$\mathrm{C}-4$ & $39.3 \mathrm{t}$ & $38.4 \mathrm{t}$ & $39.3 \mathrm{t}$ \\
$\mathrm{C}-5$ & $66.0 \mathrm{~d}$ & $70.2 \mathrm{~d}$ & $66.2 \mathrm{~d}$ \\
$\mathrm{C}-3$ & $70.4 \mathrm{~d}$ & $69.8 \mathrm{~d}$ & $71.7 \mathrm{~d}$ \\
$\mathrm{C}-2$ & $73.7 \mathrm{~d}$ & $75.6 \mathrm{~d}$ & $73.3 \mathrm{~d}$ \\
$\mathrm{C}-4^{\prime}$ & $126.1 \mathrm{~d}$ & $127.9 \mathrm{~d}$ & $126.2 \mathrm{~d}$ \\
$\mathrm{C}-3^{\prime}(2 \times)$ & $128.4 \mathrm{~d}$ & $129.7 \mathrm{~d}$ & $128.4 \mathrm{~d}$ \\
$\mathrm{C}-2^{\prime}(2 \times)$ & $129.4 \mathrm{~d}$ & $130.2 \mathrm{~d}$ & $128.9 \mathrm{~d}$ \\
$\mathrm{C}^{\prime} 1^{\prime}$ & $139.4 \mathrm{~s}$ & $137.45 . \mathrm{s}$ & $138.9 \mathrm{~s}$ \\
$\mathrm{COCH}$ & & & $170.7 \mathrm{~s}$ \\
\hline- & & &
\end{tabular}

a In ppm downfield of TMS at ambient room temperature.

- In $\mathrm{CDCl}_{3}$.

In $\mathrm{CD}_{3} \mathrm{COOD}$

Assignments may be interchanged.
Table 2. ${ }^{1} \mathrm{H}$ NMR assignments of $\mathrm{L}-657,398$ (1) in $\mathrm{CD}_{3} \mathrm{COOD}^{\mathrm{a}}$.

\begin{tabular}{lll}
\hline $\begin{array}{c}\text { Assign- } \\
\text { ment }\end{array}$ & \multicolumn{1}{c}{1} & \multicolumn{1}{c}{ Comments } \\
\hline $1-\mathrm{H}_{\mathrm{a}}$ & $3.14 \mathrm{dd}(5,13.5)$ & $\begin{array}{l}J_{1 \mathrm{a}, \mathrm{2}}=5, \\
\end{array}$ \\
$1-\mathrm{H}_{\mathrm{b}}$ & $3.29 \mathrm{dd}(10,13.5)$ & $J_{1 \mathrm{a}, \mathrm{b}}=13.5$ \\
$2-\mathrm{H}$ & $3.47 \mathrm{dt}(10, \sim 4.5)$ & $J_{1 \mathrm{~b}, 2}=10$ \\
$3-\mathrm{H}$ & $4.33 \mathrm{ddd}(1.5,4.3,6.8)$ & $J_{2,3}=4.3$, \\
& & $J_{3,4 \mathrm{~b}}=6.8$, \\
& & $J_{3,4 \mathrm{a}}=1.5$ \\
$4-\mathrm{H}_{\mathrm{a}}$ & $1.93(1.5,7.2,14.8)$ & $J_{4 \mathrm{a}, 5}=7.2$, \\
& & $J_{4 \mathrm{a}, 4 \mathrm{~b}}=14.8$ \\
$4-\mathrm{H}_{\mathrm{b}}$ & $2.65 \mathrm{ddd}(6.8,9.8,14.8)$ & $J_{4 \mathrm{~b}, 5}=9.8$ \\
$5-\mathrm{H}$ & $\sim 3.31 \mathrm{obsc}$ & \\
$6-\mathrm{H}_{\mathrm{a}}$ & $\sim 2.03 \mathrm{~m}$ & \\
$6-\mathrm{H}_{\mathrm{b}}$ & $\sim 1.76 \mathrm{~m}$ & \\
$\left(\mathrm{CH}_{2}\right)_{7}$ & $\sim 1.28 \mathrm{~m}$ & \\
$\mathrm{CH}_{3}$ & $0.88 \mathrm{t}(6.8)$ & \\
\hline
\end{tabular}

a Chemical shifts are in ppm downfield of TMS using the solvent peak at $\delta 2.03$ as reference. Coupling constants in $\mathrm{Hz}$ are given in parentheses.

obsc: Obscured (overlapping signals).

Fig. 1. Partial structures $\mathbf{A}$ and $\mathbf{B}$.

$\begin{array}{cc}\mathrm{CH}_{3}\left(\mathrm{CH}_{2}\right)_{\mathrm{n}^{-}} & \mathrm{PhCH}_{2} \mathrm{CH}(\mathrm{X}) \mathrm{CH}(\mathrm{OH}) \mathrm{CH}_{2} \mathrm{CH}(\mathrm{X}) \mathrm{CH}_{2}- \\ \mathbf{A} & \text { B }\end{array}$

\section{Results and Discussion}

Structure of L-657,398

HR-MS of the compound gave an empirical formula of $\mathrm{C}_{21} \mathrm{H}_{35} \mathrm{NO}$ and forms a monoacetate derivative $\mathrm{C}_{23} \mathrm{H}_{37} \mathrm{NO}_{2}$ on acetylation. The molecular formula was confirmed by ${ }^{13} \mathrm{C}$ NMR analysis including $\mathrm{APT}^{7)}$ spectra in $\mathrm{CDCl}_{3}$, which indicated 21 carbons comprising $1 \times \mathrm{CH}_{3}, 10 \times$ $\mathrm{CH}_{2}, 1 \times \mathrm{CH}_{3} \mathrm{~N}, 3 \times \mathrm{CHX}(\mathrm{X}=\mathrm{O}$ or $\mathrm{N}), 5 \times \mathrm{CH}=$ and $1 \times \mathrm{C}=$ (see Table 1) implicating 34 carbon bound protons. ${ }^{13} \mathrm{C}$ NMR assignments were

Fig. 2. The structure of L-657,398 (1), L-657,398 monoacetate (2) and anisomycin (3).

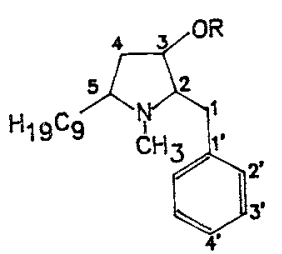

$\begin{array}{ll}\text { L-657,398 (1) } & \mathrm{R}=\mathrm{H} \\ \text { L-657,398 mono- } & \mathrm{R}=\mathrm{Ac} \\ \text { acetate (2) } & \end{array}$<smiles>COc1ccc(C[C@H]2NC[C@@H](O)[C@H]2OC(C)=O)cc1</smiles>

Anisomycin (3) made based on a HETCOR experiment in $\mathrm{CD}_{3} \mathrm{COOD}$ (Table 1). The molecule has 5 units of unsaturation and/or rings and must, therefore, have an extra ring in addition to the phenyl ring suggested by the ${ }^{13} \mathrm{C}$ NMR data. ${ }^{1} \mathrm{H}-{ }^{-1} \mathrm{H}$ connectivity experiments, including $1 \mathrm{D}$ double irradiation and $2 \mathrm{D}$ correlation spectroscopy $(\mathrm{COSY})$ were carried out in a variety of solvents $\left(\mathrm{CDCl}_{3}\right.$, benzene- $d_{k}$, 
Table 3. Activity of L-657,398 and anisomycin against a panel of bacteria, filamentous fungi, and yeasts.

\begin{tabular}{|c|c|c|c|c|c|c|c|c|c|c|c|c|c|c|c|c|}
\hline \multirow{3}{*}{$\begin{array}{l}\text { Culture } \\
\text { number }\end{array}$} & \multirow{3}{*}{ Media $^{b}$} & \multirow{3}{*}{$\begin{array}{l}\text { Temp } \\
\left({ }^{\circ} \mathrm{C}\right)\end{array}$} & \multirow{3}{*}{ Strain designation } & \multicolumn{12}{|c|}{ Zone diameter ${ }^{2}(\mathrm{~mm})$} & \\
\hline & & & & \multicolumn{6}{|c|}{ Anisomycin $(\mu \mathrm{g} / \mathrm{ml})$} & \multicolumn{7}{|c|}{$\mathrm{L}-657,398(\mu \mathrm{g} / \mathrm{ml})$} \\
\hline & & & & 1,000 & 500 & 250 & 125 & 62 & 31 & 1,000 & 500 & 250 & 125 & 62 & 31 & 16 \\
\hline MA4798 & YED & 28 & Streptomyces sp. & 0 & 0 & 0 & 0 & 0 & 0 & $13 \mathrm{~S}$ & $12 \mathrm{H}$ & $10 \mathrm{~V}$ & 0 & 0 & 0 & 0 \\
\hline MY34 & YED & 28 & Saccharomyces cerevisiae & $25 F$ & $22 \mathrm{~F}$ & $20 \mathrm{~F}$ & $16 \mathrm{~F}$ & $14 \mathrm{~S}$ & $11 \mathrm{~S}$ & $20 \mathrm{~S}$ & $17 \mathrm{~S}$ & $15 S$ & $11 \mathrm{~S}$ & $9 \mathrm{H}$ & 0 & 0 \\
\hline MY992 & YED & 28 & Candida albicans & $11 \mathrm{~S}$ & $8 \mathrm{H}$ & 0 & 0 & 0 & 0 & $12 \mathrm{H}$ & $10 \mathrm{H}$ & $9 \mathrm{~V}$ & 0 & 0 & 0 & 0 \\
\hline MF4626 & PDA & 28 & Cochliobolus miyabeanus & $17 \mathrm{~F}$ & $13 \mathrm{~F}$ & $10 \mathrm{H}$ & $8 \mathrm{~V}$ & 0 & 0 & $40 \mathrm{~F}$ & $36 \mathrm{~S}$ & $32 \mathrm{~S}$ & $31 \mathrm{~s}$ & $27 \mathrm{~S}$ & $21 \mathrm{~S}$ & $17 \mathrm{~S}$ \\
\hline MF442 & YED & 28 & Aspergillus niger & $8 \mathrm{~V}$ & 0 & 0 & 0 & 0 & 0 & $13 \mathrm{~S}$ & $10 \mathrm{H}$ & $8 \mathrm{~V}$ & 0 & 0 & 0 & 0 \\
\hline MF5014 & PDA & 28 & Penicillium sp. & $23 \mathrm{~F}$ & $18 \mathrm{~F}$ & $14 \mathrm{~F}$ & $11 \mathrm{H}$ & $9 \mathrm{~V}$ & 0 & $11 \mathrm{H}$ & $8 \mathrm{~V}$ & 0 & 0 & 0 & 0 & 0 \\
\hline MF5016 & PDA & 28 & Penicillium sp. & $23 \mathrm{~F}$ & $17 \mathrm{~F}$ & $14 S$ & $10 \mathrm{~S}$ & $9 \mathrm{H}$ & 0 & $9 \mathrm{H}$ & $7 \mathrm{~V}$ & 0 & 0 & 0 & 0 & 0 \\
\hline MF5020 & PDA & 28 & Penicillium sp. & $13 \mathrm{H}$ & $9 \mathbf{H}$ & 0 & 0 & 0 & 0 & $7 \mathrm{~V}$ & 0 & 0 & 0 & 0 & $\mathbf{0}$ & 0 \\
\hline MF11 & PDA & 28 & A. niger & $14 \mathrm{~S}$ & $10 \mathrm{H}$ & 0 & 0 & 0 & 0 & $12 S$ & $10 \mathrm{~S}$ & $8 \mathrm{H}$ & 0 & 0 & 0 & 0 \\
\hline MF4064 & PDA & 28 & Trichoderma sp. & $10 \mathrm{~V}$ & $8 \mathrm{~V}$ & 0 & 0 & $\mathbf{0}$ & 0 & $9 \mathrm{H}$ & $7 \mathrm{~V}$ & 0 & 0 & 0 & $\mathbf{0}$ & 0 \\
\hline MF4332 & PDA & 28 & Phoma sp. & 0 & 0 & 0 & 0 & 0 & 0 & $13 \mathrm{~S}$ & $9 \mathrm{H}$ & 0 & 0 & 0 & $\mathbf{0}$ & 0 \\
\hline MF3560 & PDA & 28 & Trichoderma lignorum & $9 \mathrm{~V}$ & $7 \mathrm{~V}$ & 0 & 0 & 0 & $\mathbf{0}$ & $19 \mathrm{H}$ & $16 \mathrm{H}$ & $13 \mathrm{~V}$ & 0 & $\mathbf{0}$ & 0 & 0 \\
\hline MF4014 & PDA & 28 & Fusarium oxysporum & $9 \mathrm{H}$ & $8 \mathrm{~V}$ & $8 \mathrm{~V}$ & 0 & 0 & 0 & 0 & 0 & 0 & 0 & 0 & 0 & 0 \\
\hline MF1996 & PDA & 28 & Ustilago zeae & $22 \mathrm{H}$ & $19 \mathrm{H}$ & $14 \mathrm{H}$ & $10 \mathrm{H}$ & $8 \mathrm{~V}$ & 0 & $27 \mathrm{~S}$ & $24 S$ & $20 \mathrm{~S}$ & $17 \mathrm{~S}$ & $10 \mathrm{H}$ & $8 \mathrm{H}$ & 0 \\
\hline MF4042 & PDA & 28 & Ceratocystis ulmi & $10 \mathrm{~s}$ & $9 \mathbf{H}$ & $8 \mathrm{~V}$ & 0 & 0 & 0 & $23 \mathrm{~F}$ & $20 \mathrm{~S}$ & $17 \mathrm{H}$ & $14 \mathrm{H}$ & $8 \mathrm{~V}$ & 0 & 0 \\
\hline MF3550 & PDA & 28 & Alternaria solani & 0 & 0 & 0 & 0 & 0 & 0 & $32 \mathrm{~F}$ & $30 \mathrm{~F}$ & $29 \mathrm{~S}$ & $28 \mathrm{H}$ & $19 \mathrm{H}$ & $18 \mathrm{H}$ & $13 \mathrm{H}$ \\
\hline MF3794 & PDA & 28 & Verticillium serrae & 0 & 0 & 0 & 0 & 0 & 0 & $21 \mathrm{~F}$ & $17 \mathrm{~S}$ & $13 \mathrm{H}$ & $10 \mathrm{H}$ & 0 & 0 & 0 \\
\hline MF3587 & PDA & 28 & Botrytis allii & $26 \mathrm{~S}$ & $22 \mathrm{~S}$ & $17 \mathrm{H}$ & $12 \mathrm{H}$ & $10 \mathrm{~V}$ & 0 & & & & & & & \\
\hline MF3769 & PDA & 28 & Scopulariopsis communis & 0 & 0 & 0 & 0 & 0 & 0 & 0 & 0 & 0 & 0 & 0 & 0 & 0 \\
\hline MF4641 & PDA & 28 & Cephalosporium sp. & 0 & 0 & 0 & 0 & 0 & 0 & $9 \mathrm{H}$ & $8 \mathrm{~V}$ & 0 & 0 & 0 & 0 & 0 \\
\hline MF4608 & PDA & 28 & Cercospora beticola & $10 \mathrm{H}$ & $8 \mathrm{~V}$ & 0 & 0 & 0 & 0 & $20 \mathrm{H}$ & $18 \mathrm{H}$ & $15 \mathrm{H}$ & $12 \mathrm{H}$ & $12 \mathrm{~V}$ & $8 \mathrm{~V}$ & 0 \\
\hline MY1012 & SDA & 37 & Candida tropicalis & $30 \mathrm{~F}$ & $26 \mathrm{~F}$ & $22 \mathrm{~F}$ & $15 S$ & $11 \mathrm{H}$ & $9 \mathrm{~V}$ & $25 \mathrm{~F}$ & $21 \mathrm{~F}$ & $18 \mathrm{~F}$ & $15 S$ & $11 \mathrm{~S}$ & $8 \mathrm{H}$ & 0 \\
\hline MY1022 & SDA & 37 & Candida rugosa & $9 \mathrm{~V}$ & 0 & 0 & 0 & 0 & 0 & $8 \mathrm{H}$ & 0 & 0 & 0 & 0 & 0 & 0 \\
\hline MY1028 & SDA & 37 & C. albicans & $13 \mathrm{H}$ & $9 \mathrm{~V}$ & 0 & 0 & 0 & 0 & $10 \mathrm{H}$ & $8 \mathrm{~V}$ & 0 & 0 & 0 & 0 & 0 \\
\hline MB4558 & BHI & 37 & Acholeplasma laidlawii & & & & & & & 0 & 0 & 0 & 0 & 0 & 0 & $\mathbf{0}$ \\
\hline MY315 & SDA & 28 & Brettanomyces bruxellensis & $12 \mathrm{~V}$ & $8 \mathrm{~V}$ & 0 & 0 & 0 & 0 & 0 & 0 & 0 & 0 & 0 & 0 & $\mathbf{0}$ \\
\hline MY321 & SDA & 28 & Torulospora hansenii & $15 \mathrm{H}$ & $10 \mathrm{~V}$ & $8 \mathrm{~V}$ & 0 & 0 & 0 & $14 \mathrm{~S}$ & $12 \mathrm{~S}$ & $9 \mathrm{H}$ & 0 & 0 & 0 & $\mathbf{0}$ \\
\hline MY410 & PDA & 28 & S. cerevisiae & $21 \mathrm{~S}$ & $16 \mathrm{~S}$ & $13 S$ & $9 \mathrm{H}$ & $9 \mathrm{~V}$ & 0 & $11 \mathrm{~S}$ & $8 \mathrm{~S}$ & 0 & 0 & 0 & 0 & 0 \\
\hline MY1019 & SDA & 37 & Candida guilliermondii & 0 & 0 & 0 & 0 & 0 & 0 & $8 \mathrm{~V}$ & 0 & 0 & 0 & 0 & 0 & $\mathbf{0}$ \\
\hline MY1074 & SDA & 28 & Cryptococcus laurentii & $17 \mathrm{~V}$ & $12 \mathrm{~V}$ & 0 & 0 & 0 & 0 & $22 \mathrm{~S}$ & $17 \mathrm{~S}$ & $15 S$ & $11 \mathrm{~S}$ & 0 & 0 & $\mathbf{0}$ \\
\hline MY1099 & SDA & 37 & C. albicans & $10 \mathrm{~V}$ & $8 \mathrm{~V}$ & 0 & 0 & 0 & 0 & $9 \mathrm{~V}$ & 0 & 0 & 0 & 0 & 0 & 0 \\
\hline MY1100 & SDA & 37 & Candida pseudotropicalis & $21 \mathrm{~F}$ & $16 \mathrm{~F}$ & $13 \mathrm{~F}$ & $9 \mathrm{H}$ & 0 & 0 & $16 \mathrm{~S}$ & $13 \mathrm{~S}$ & $8 \mathrm{~S}$ & $7 \mathrm{~V}$ & 0 & 0 & 0 \\
\hline MY1062 & SDA & 37 & Torulopsis glabrata & $8 \mathbf{H}$ & 0 & 0 & 0 & 0 & 0 & 0 & 0 & 0 & 0 & 0 & 0 & 0 \\
\hline MY1070 & SDA & 28 & Cryptococcus albidus & 0 & 0 & 0 & 0 & 0 & 0 & $12 \mathrm{H}$ & $9 \mathrm{~V}$ & 0 & 0 & 0 & 0 & 0 \\
\hline MY1073 & SDA & 28 & Cryptococcus laurentii & $14 \mathrm{~V}$ & $9 \mathrm{~V}$ & 0 & 0 & 0 & 0 & $19 \mathrm{H}$ & $15 \mathrm{H}$ & $13 \mathrm{H}$ & $8 \mathrm{~V}$ & 0 & 0 & 0 \\
\hline MY1077 & SDA & 28 & C. laurentii & $8 \mathrm{~V}$ & 0 & 0 & 0 & 0 & 0 & $17 \mathrm{H}$ & $15 \mathrm{H}$ & $12 \mathrm{H}$ & $8 \mathrm{~V}$ & 0 & 0 & 0 \\
\hline MY1113 & SDA & 28 & Kluyveromyces fragilis & $14 \mathrm{~F}$ & $10 \mathrm{~S}$ & $9 \mathrm{H}$ & $8 \mathrm{~V}$ & 0 & 0 & $14 S$ & $10 S$ & $8 \mathrm{H}$ & 0 & 0 & 0 & 0 \\
\hline MY1029 & $\mathrm{SDA}$ & 28 & C. albicans & $9 \mathrm{~V}$ & 0 & 0 & 0 & 0 & 0 & $8 \mathrm{~V}$ & 0 & 0 & 0 & 0 & 0 & 0 \\
\hline MY1055 & SDA & 28 & C. albicans & $8 \mathrm{~V}$ & 0 & 0 & 0 & 0 & 0 & $9 \mathrm{~V}$ & $8 \mathrm{~V}$ & 0 & 0 & 0 & 0 & 0 \\
\hline MF4784 & PDA & 37 & Rhizomucor miehei & 0 & 0 & 0 & 0 & 0 & 0 & $9 \mathrm{H}$ & $7 \mathrm{~V}$ & 0 & 0 & 0 & 0 & 0 \\
\hline MF383 & PDA & 28 & Aspergillus flavus & 0 & 0 & $\mathbf{0}$ & 0 & 0 & 0 & $15 \mathrm{~S}$ & $12 \mathrm{~S}$ & $9 \mathbf{H}$ & 0 & 0 & $\mathbf{0}$ & 0 \\
\hline MF4839 & PDA & 28 & Aspergillus fumigatus & $8 \mathrm{~V}$ & 0 & 0 & 0 & 0 & 0 & $14 S$ & $8 \mathrm{H}$ & 0 & 0 & 0 & 0 & 0 \\
\hline
\end{tabular}

:Fuzzy edge, $\mathrm{S}$ : slightly hazy, $\mathrm{H}$ : haz

b Difco PDA: Potato dextrose agar, SDA: SABourAuD dextrose agar, YED: yeast extract dextrose, BHI; brain heart infusion. 
$\mathrm{CD}_{3} \mathrm{COOD}$ ) of which $\mathrm{CD}_{3} \mathrm{COOD}$ proved to be optimal in terms of chemical shift dispersion.

The data (see Table 2) suggested partial sequences A and B (Fig. 1) which on the basis of the empirical formula and ${ }^{13} \mathrm{C}$ NMR data can be formulated into the $N$-methyl pyrrolidine structure 1 (Fig. 2). The ${ }^{1} \mathrm{H}$ NMR spectrum of the acetate derivative 2 confirms the assignment of the $\mathrm{OH}$ group by the characteristic downfield shift of 3-H from $\delta 4.33$ to 5.13. Confirmation was obtained by MS fragmentation which is directed by the ring nitrogen, resulting in a base peak at $\mathrm{m} / \mathrm{z} 226.2165$ (calcd for $\left.\mathrm{C}_{14} \mathrm{H}_{28} \mathrm{NO}, m / z 226.2170\right)$ due to loss of tropylium ion $(\mathrm{m} / z$ 91) and a weaker ion at $\mathrm{m} / \mathrm{z} 190.1224$ (calcd for $\mathrm{C}_{12} \mathrm{H}_{18} \mathrm{NO}, \mathrm{m} / z$ 190.1231) corresponding to the loss of the $\mathrm{C}_{9}$ alkyl side chain. The corresponding ions in the $O$-acetate derivative 2 are greater by 42 mass units. The MS, ${ }^{1} \mathrm{H}$ NMR (see Table 2) and ${ }^{13} \mathrm{C}$ NMR (see Table 1) are, therefore, fully consistent with the pyrrolidine structure 1 for L-657,398 shown in Fig. 2. However, the relative stereochemistry could not be unambiguously assigned based on ${ }^{1} \mathrm{H}$ NMR.

\section{Antifungal Activity}

The antifungal activity of L-657,398 was determined by the disk diffusion method (as described in the Materials and Methods section) and compared to anisomycin at a variety of concentrations ranging from $1 \mathrm{mg} / \mathrm{ml}$ to $16 \mu \mathrm{g} / \mathrm{ml}$. The results of this evaluation indicated that the antifungal spectrum of L-657,398 was broad, with activity against both filamentous fungi and yeasts, and significantly broader than anisomycin.

\section{References}

1) Turner, W. B. \& D. C. Aldridge: In Fungal Metabolites. II. pp. 87, 90, 128, 164, 198, 316, 416, Academic Press, London, 1983

2) Sorin, B. A. \& F. W. TANner, Jr.: Anisomycin, a new antiprotozoan antibiotic. J. Am. Chem. Soc. 76: 4053,1954

3) Tanner, F. W., Jr.; B. A. Sobin \& J. F. GArdocki: Some chemical and biologic properties of anisomycin. In Antibiotics Annual 1954-1955. Eds., H. Welch \& F. Marti-Ibañez, pp. 809 812, Medical Encyclopedia, Inc., New York, 1955

4) Jimenez, A. \& D. VÁzquez: Mechanism of action of antieukaryotic and antiviral compounds. In Antibiotics. Volume V/Part 2. Ed., F. E. HAHN, pp. 1 19, Springer-Verlag, Berlin, 1979

5) Grolloman, A. P.: Inhibitors of protein biosynthesis. II. Mode of action of anisomycin. J. Biol. Chem. 242: 3226 3233, 1967

6) Budzikiewicz, H.; C. Djerassi \& D. H. Williams: In Mass Spectrometry of Organic Compounds. pp. $309 \sim 320$, Holden-Day, Inc., San Francisco, 1967

7) Patt, S. L. \& J. N. Schoolery: Attached proton test for carbon-13 NMR. J. Org. Mag. Res. 46: 535 539,1982 